\title{
Crisis-Checklisten für die Endoskopie und den OP
}

\author{
Tobias Schulze $^{\mathrm{a}}$ Sören Runge ${ }^{\mathrm{b}} \quad$ Martin von der Heyden ${ }^{\mathrm{c}}$ \\ ${ }^{\text {a} A b t e i l u n g ~ f u ̈ r ~ A l l g e m e i n e ~ C h i r u r g i e, ~ V i s z e r a l-, ~ T h o r a x-~ u n d ~ G e f a ̈ ß c h i r u r g i e, ~ K l i n i k ~ u n d ~ P o l i k l i n i k ~ f u ̈ r ~ C h i r u r g i e, ~}$

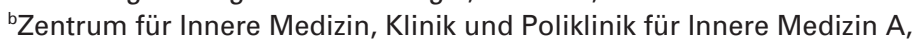 \\ ${ }^{\mathrm{c}}$ Klinik und Poliklinik für Anästhesiologie und Intensivmedizin, Universitätsmedizin Greifswald, Deutschland
}

\section{Schlïsselwörter \\ Crisis-Checkliste $\cdot$ Notfall · Endoskopie $\cdot$ Chirurgie}

\section{Zusammenfassung}

Hintergrund: Im Vergleich zu präventiven Sicherheitschecklisten ist der Nutzen von Crisis-Checklisten im OP und in der Endoskopie außerhalb von anästhesiologischen Fragestellungen erst sehr wenig untersucht. Die verfügbaren klinischen Daten werden im Folgenden ausgewertet und Aspekte aus anderen sicherheitsrelevanten Bereichen sowie aus den Verhaltenswissenschaften werden diskutiert. Methode: Pubmed-Suche anhand der Stichwörter "Crisis Checklist» oder "Emergency Checklist" - kombiniert mit "Surgery" oder "Endoscopy" und unter Ausschluss anästhesiologischer Fragestellungen. Ergebnisse: Trotz zahlreicher theoretischer Anhaltspunkte aus der Kognitions- und Gruppenpsychologie, der Stressforschung sowie des standardmäßigen Gebrauchs von Crisis-Checklisten in anderen sicherheitsrelevanten Bereichen (Flugwesen, Atomkraft) ist der Gebrauch von Crisis-Checklisten in der klinischen Routine nur in einer einzigen klinischen Studie außerhalb anästhesiologischer Fragestellungen erforscht. Die verfügbare Literatur weist auf einen positiven subjektiven Effekt für die Anwender und eine verbesserte Standardisierung von klinischen Abläufen in Notfallsituationen hin. Der Einfluss auf das klinische Outcome wurde nicht untersucht. Schlussfolgerungen: Klinische Daten für eine Verbesserung des Patienten-Outcomes in Notfallsituationen durch die Nutzung von Crisis-Checklisten in der Endoskopie und im OP stehen noch aus. Nur durch diese Daten können die potenziellen Nutzer von den Vorteilen des Gebrauchs von Crisis-Checklisten überzeugt und eine hohe Akzeptanz im klinischen Alltag erreicht werden. Hier ist dringend weitere klinische Forschung notwendig.

\author{
Keywords \\ Crisis checklist · Emergency · Endoscopy · Surgery
}

\section{Summary}

Use of Crisis Checklists in the Endoscopy Department and in the Operating Room

Background: In contrast to safety checklists, the routine use of crisis checklists in surgery and endoscopy departments is less established. In the following, data from available clinical trials will be reviewed and experiences from other high reliability industries and theoretical aspects from cognitive psychology will be discussed. Method: Selective search based on the keywords 'crisis checklist' or 'emergency checklist' combined with the terms 'surgery' or 'endoscopy', excluding reports with an anesthesiological focus. Results: Crisis checklists are routinely used in high reliability industries. Moreover, numerous theoretical aspects from cognitive psychology, stress research, and research on group dynamics suggest positive effects of crisis checklist usage in medical emergencies. However, extensive bibliographic search revealed only one clinical study assessing the effects of the implementation of crisis checklists in the operating room. This study suggests a positive appreciation by the user as well as positive effects on the standardization of treatment in emergency situations. However, the effect on the clinical outcome was not assessed. Conclusions: Clinical data assessing the impact of crisis checklists on the clinical outcome in emergency situations is still sparse. This data is required for the broad implementation of crisis checklists in the clinical routine in surgery and endoscopy departments. Further clinical research is absolutely essential.

\section{KARGER \\ Fax +497614520714 \\ Information@Karger.com}

www.karger.com (c) 2013 S. Karger GmbH, Freiburg

$1662-6664 / 13 / 0293-0180 \$ 38.00 / 0$

Accessible online at:

www.karger.com/vim
Dr. Dr. med. Tobias Schulze

Abteilung für Allgemeine Chirurgie, Viszeral-, Thorax- und Gefäßchirurgie

Klinik und Poliklinik für Chirurgie, Universitätsmedizin Greifswald

Ferdinand-Sauerbruch-Straße, 17475 Greifswald, Deutschland

schulzet@uni-greifswald.de 


\section{Einleitung}

Die Behandlung eines Patienten im stationären Umfeld erfordert eine Vielzahl von Prozessen unterschiedlicher, teilweise sehr hoher Komplexität, die von Angehörigen verschiedener Berufsgruppen in der korrekten zeitlichen und örtlichen Abfolge ausgeführt werden müssen. So erfordert die Versorgung eines Intensivpatienten täglich durchschnittlich 178 Handlungen [1]. An den Schnittstellen dieser Prozesse liegen häufig Fehlerquellen verborgen, die zu einer erhöhten Patientenmorbidität und -mortalität durch hier eingetretene Komplikationen führen. Diese stellen aufgrund ihrer Häufigkeit ein nicht zu vernachlässigendes sozioökonomisches Problem dar: Etwa einer von 150 Krankenhauspatienten verstirbt infolge von Komplikationen [2]. In den USA sind Komplikationen im Rahmen medizinischer Behandlungen für mehr Todesfälle verantwortlich als Verkehrsunfälle, Brustkrebs oder AIDS-Erkrankungen [3]. Komplikationen im Rahmen einer Operation sind dabei die weitaus häufigsten [2]. Im Jahr 2006 wurde von der World Health Organization (WHO) eine OP-Sicherheitscheckliste mit dem Ziel eingeführt, die Patientensicherheit im OP durch die Vermeidung von Schnittstellenfehlern und die Förderung der Standardisierung von Abläufen zu erhöhen. Der Erfolg dieser Maßnahme im chirurgischen Routinebetrieb wurde bereits von zahlreichen Autoren nachgewiesen [4, 5]. Im Gegensatz dazu liegen nur wenige Daten zum klinischen Nutzen von Crisis-Checklisten in Notfallsituationen im Krankenhaus vor [6]. Dahingegen ist deren Gebrauch in der Luftfahrt oder in Hochrisikoindustrien längst Routine.

Notfallsituationen im medizinischen Bereich sind im Vergleich zur elektiven Behandlung von Patienten mit einer ganzen Reihe von Umständen verbunden, die die Behandlung des individuellen Patienten erheblich erschweren (Tab. 1) [7]. In der Chirurgie und in endoskopischen Abteilungen handelt es sich dabei meist um Notfälle, die mit einem kardialen oder respiratorischen Versagen sowie schweren Blutungen einhergehen. Warum und in welcher Weise Crisis-Checklisten zur Prozessoptimierung in der Versorgung medizinischer Notfälle beitragen können oder könnten, soll im Folgenden aufgezeigt werden. Hierzu wurde eine selektive Literaturrecherche in Pubmed und Google mit den Stichwörtern «Crisis Checklist» und «Emergency Checklist» - kombiniert mit «Surgery» oder «Endoscopy» und unter Ausschluss anästhesiologischer Fragestellungen - durchgeführt.

\section{Gebrauch von Checklisten in Notfallsituationen: Anforderungen an die Crisis-Checkliste}

Bei der OP-Sicherheitscheckliste der WHO handelt es sich um eine typische statisch sequenzielle Checkliste mit konsekutiver Bestätigung und Kontrolle, mittels derer das Vorhandensein von für die geplante Operation notwendigen Infor-
Tab. 1. Die Versorgung von Notfallpatienten erschwerende Faktoren

Personen- und persönlichkeitsbedingte Faktoren

Stressbedingte Fehler

Unfähigkeit, sich in das versorgende Team zu integrieren

Teambedingte Faktoren

Schwierigkeiten bei der Maßnamenkoordination

Schlechte Kommunikation

Fehlendes Benennen klarer Ziele und aktueller Schwierigkeiten

Zusammenarbeit von Teammitgliedern unterschiedlicher professioneller

Sozialisation und unterschiedlichen Ausbildungsstands

Unwilligkeit, das falsche Handeln Ranghöherer infrage zu stellen

Situationsbedingte Faktoren

Unvollständige Informationen über Anamnese

Häufige Unterbrechungen

Verminderte Vigilanz und Müdigkeit, z.B. nachts

mationen und wesentlichen technischen Voraussetzungen verifiziert wird. An Crisis-Checklisten für Notfallsituationen werden eine Reihe von zusätzlichen Anforderungen gestellt, die den Besonderheiten einer durch einen erheblichen Zeitdruck und eine hohe psychische Belastung der beteiligten Teammitglieder geprägten Situation Rechnung tragen müssen. Meist handelt es sich bei ihnen um dynamische Checklisten, die sich Flussdiagrammen bedienen, um eine komplexe Entscheidungsfindung zu leiten.

Grundvoraussetzung für den Gebrauch einer Crisis-Checkliste ist die korrekte Symptominterpretation durch die beteiligten Teammitglieder, die zur Wahl der in der konkreten Notfallsituation adäquaten Crisis-Checkliste führt [8]. Idealerweise sollte eine Crisis-Checkliste interne Kontroll-Items enthalten, die in nicht eindeutigen Notfallsituationen die Wahl der korrekten Checkliste erleichtern.

Der Einsatz von Crisis-Checklisten im klinischen Alltag erfordert weiterhin, dass die beteiligten Mitarbeiter ausreichend über die Existenz der Checklisten, deren Benennung und deren Auffindbarkeit informiert sind. Ein und dieselbe Notfallsituation kann durch verschiedene Termini charakterisiert werden, z.B. Dyspnoe, Atemnot und respiratorisches Versagen. Das Ablegen einer Crisis-Checkliste unter mehreren Stichworten kann in elektronischen Versionen einer Checkliste $\mathrm{zu}$ deren schnellerem Auffinden in Notfallsituationen führen.

Ein weiterer limitierender Faktor für den Einsatz von Crisis-Checklisten ist der Zeitmangel in einer Notfallsituation. Der klinische Zustand des Patienten kann sich in einer kürzeren Zeitspanne ändern, als die von der Checkliste vorgeschlagenen Maßnahmen zu ihrer vollständigen Ausführung erfordern. Dieses Phänomen ist auch in der im Umgang mit Checklisten wesentlich routinierteren Luftfahrt $\mathrm{zu}$ beobachten: «The ... events took place over a time span of less than 4 minutes during a critical phase of flight ... the events occurred simultaneously with radio transmissions, configuration changes, airspeed changes and constantly changing altitude ... 
What we learned from this event is that running the emergency checklists may not be a classical situation where one has plenty of time for analysis and application of curative measures» [9]. In einem anderen Fall aus dem Jahr 1998 hätte die komplette Ausführung der in der konkreten Situation adäquaten Rauch- und Feuer-Checklisten des Fluges Swissair 111 ungefähr 30 min in Anspruch genommen [10]. Zwischen dem ersten Bemerken von Brandgeruch und dem Absturz des Fluges lagen aber letztendlich nur ca. 20 min. Diese Beispiele zeigen, dass im Vergleich zur WHO-Sicherheitscheckliste dem Zeitfaktor bei der Erstellung von Crisis-Checklisten eine weitaus größere Bedeutung zukommt. Da Crisis-Checklisten komplexe medizinische Situationen adäquat und auch leitliniengerecht widerspiegeln müssen, kann dem Zeitfaktor aber nur bis zu einem bestimmten Punkt Rechnung getragen werden. Hier ist ein regelmäßiges situatives Training des Umgangs mit den Checklisten beispielsweise in Notfallsimulatoren unerlässlich.

Ein weiteres, insbesondere für Crisis-Checklisten wesentliches Merkmal ist die eindeutige Verständlichkeit der empfohlenen Maßnahmen für den Anwender. Der empfohlene Schritt muss für das ausführende Teammitglied eindeutig benannt sein und dem Ausbildungsstand des Teammitglieds entsprechen. Auch hier wird deutlich, wie wichtig die Einbeziehung aller an der medizinischen Versorgung Beteiligten (Ärzte, Pflegekräfte, medizinisch-technische Assistenten, Rettungssanitäter) in die Checklistenerstellung ist, um im Checklistendesign eine Verständlichkeit über die Ausbildungsgrenzen hinweg zu erreichen.

Zahlreiche Untersuchungen haben gezeigt, dass die kognitiven Fähigkeiten der unter akuter Stressbelastung Handelnden häufig eingeschränkt sind. Diesem Umstand muss bei der Erstellung der häufig in zeitkritischen Situationen auszuführenden Crisis-Checklisten Rechnung getragen werden. Dazu gehören beispielsweise das Vermerken der zu applizierenden Medikamentendosen, Tubusgrößen bei der Versorgung pädiatrischer Patienten usw. Auch hier wird deutlich, welche Anforderungen sich an eine Crisis-Checkliste im Spannungsfeld zwischen notwendiger Kürze und Prägnanz in zeitkritischen Situationen einerseits und der Lieferung ausreichender Information in psychischen Ausnahmesituationen andererseits ergeben.

\section{Crisis-Checklisten zur Fehlervermeidung in stressbelasteten Situationen}

Der Begriff «Stress» wurde ursprünglich von dem Endokrinologen Hans Selye in den 30er-Jahren des letzten Jahrhunderts geprägt [11]. Die initiale Stressantwort resultiert dabei aus den Anforderungen, die von der Umwelt auf ein Individuum projiziert werden, und dessen Ressourcen - extern und intern -, diesen Anforderungen auch gerecht zu werden. Wenn ein Individuum die ihm zur Verfügung stehenden Mög- lichkeiten als der antizipierten oder tatsächlichen Herausforderung adäquat empfindet, wird es den Stressor eher als Herausforderung und damit Eustress wahrnehmen. Wird die Situation dagegen als die eigenen Ressourcen übersteigend wahrgenommen, resultiert hieraus Angst und Disstress mit allen damit verbundenen Einflüssen auf die individuelle kognitive und physische Leistungsfähigkeit, aber auch auf die kollektive Leistungsfähigkeit im Team.

Untersuchungen zum Einfluss von Stress auf die Informationswahrnehmung haben unterschiedliche Ergebnisse erbracht (für eine Übersicht siehe [12]). Es erscheint aber sicher, dass Stress in Situationen, die die Integration von Informationen aus verschiedenen Quellen erfordern, wie dies in Notfallsituationen der Fall ist, zu einer deutlichen Einschränkung der kognitiven Fähigkeiten führt [13-15]. Das Überdenken einmal getroffener Entscheidungen und Diagnosen wird in diesem Setting unwahrscheinlicher [16].

Stress hat auch beträchtliche Auswirkungen auf die Funktion des Gedächtnisses. Während moderate Stresslevel einen eher günstigen Einfluss auf die Konsolidierung von Gedächtnisinhalten haben, wird die Leistungsfähigkeit des Arbeitsgedächtnisses durch Stress erheblich eingeschränkt $[17,18]$. Doch gerade an dieses werden in medizinischen Notfallsituationen besondere Anforderungen gestellt: Das kurzzeitige Erinnern der anamnestischen Informationen des Patienten, der von den Überwachungsmonitoren angezeigten Vitalparameter, der Ergebnisse der Notfalldiagnostik und der Informationen über bereits erfolgte Therapieschritte sowie die Integration der erhaltenen Informationen stellen eine außergewöhnliche Herausforderung für das Arbeitsgedächtnis dar. Auch der Abruf von Gedächtnisinhalten wie etwa Medikamentendosierungen ist unter Stress deutlich erschwert [19].

Ein Entscheidungsprozess unter Stressbedingungen unterscheidet sich deutlich von einer unter normalen Bedingungen erfolgenden Entscheidungsfindung. Während Letztere zunächst mit einer geordneten Informationssuche, deren Abwägung und dem Durchspielen verschiedener Entscheidungsalternativen und deren Konsequenzen einhergeht, sind Entscheidungen in Notfallsituationen durch eine gesteigerte Impulsivität und ungeordnete Informationsverarbeitung gekennzeichnet [20].

Aus den geschilderten Veränderungen der kognitiven Fähigkeiten ist leicht herzuleiten, dass eine Crisis-Checkliste in stressbeladenen Notfallsituationen ein geeignetes Werkzeug darstellt, den dargestellten negativen Stressauswirkungen effektiv entgegenzutreten. Durch das Verzeichnen von Flussdiagrammen und den zugehörigen Entscheidungskriterien kann das Erheben notwendiger Informationen systematisiert und Entscheidungsfindungen in Notfallsituationen können rationalisiert werden. Das Unterlassen differentialdiagnostischer Abwägungen oder notwendiger Untersuchungen ist als Fehlerursache viermal so häufig wie aktive falsche Handlungen [21]. Checklisten können «Fehler durch Unterlassen» re- 
duzieren. Durch das Darstellen beispielsweise der Posologien der essenziellen Notfallmedikamente können mnestische Einschränkungen ausgeglichen werden.

\section{Checklisten zur Implementierung des neuesten wissenschaftlichen Standes bei Notfallsituationen}

Medizinisches Wissen erfährt seit Jahren eine rasante Entwicklung. Richtlinien und Empfehlungen werden durch Fachgesellschaften regelmäßig aktualisiert und sind einer dynamischen Entwicklung mit teilweise kurzen Halbwertszeiten unterworfen.

Crisis-Checklisten sind eine Möglichkeit, neue Erkenntnisse und Empfehlungen durch regelmäßige Aktualisierung und häufiges Mitarbeitertraining für Notfallsituationen auf kurzem Weg in die Klinik einzuführen.

\section{Checklisten zur Verbesserung des Zusammenspiels im Team}

Notfallsituationen erfordern das geordnete und koordinierte Handeln einer Gruppe, die sich aus Mitgliedern ganz unterschiedlichen Qualifikationsniveaus und unterschiedlicher Berufserfahrung zusammensetzt. Eine Studie über Fehlerursachen bei der Versorgung von Intensivpatienten zeigte, dass eine schlechte Kommunikation zwischen Krankenschwestern und ärztlichem Personal für bis zu 37\% aller Fehler ursächlich war [22]. Die Einflüsse von Stress auf das Zusammenspiel und die Struktur in einer solch heterogenen Gruppe wurden im medizinischen Sektor wenig untersucht. Zusätzlich bestehen häufig berufsgruppenspezifische Herangehensweisen an spezielle Probleme, deren Wurzeln unter anderem in einer unterschiedlichen Ausbildung und professionellen Sozialisation zu suchen sind. Gerade konkrete, die interdisziplinäre Zusammenarbeit erfordernde Situationen lassen diese Unterschiede zutage treten. In einer Studie an norwegischen Luftrettungsteams konnte gezeigt werden, das Rettungssanitäter und Piloten feste Regeln und Handlungsabläufe wesentlich mehr schätzen als Mediziner, die Flexibilität und die Auswahlmöglichkeit aus mehreren Optionen als wichtig einschätzten [23].

In diesem Spannungsfeld konnte gezeigt werden, dass das Vorhandensein einer «impliziten Koordination» (implicit coordination) eine wesentliche Voraussetzung für ein erfolgreiches Agieren der Gruppe in einer Stresssituation darstellt [24]. Hierunter verstanden Cannon-Bowers und Salas [24] ein gemeinsames Verständnis der aktuellen Situation, eine gemeinsame korrekte Interpretation der auftretenden Ereignisse sowie eine weitgehende Antizipation potenzieller Probleme, bald notwendig werdender Handlungen sowie notwendiger Ressourcen. Diese «implizite Koordination» erfordert eine gemeinsame «mentale»
Modellvorstellung der Situation und der daraus resultierenden Anforderungen.

In Kombination mit einem intensiven Teamtraining von Notfallsituationen können Crisis-Checklisten die Entstehung einer «impliziten Koordination» fördern. Im Gegensatz zum Einfluss von Simulatortraining ist die Auswirkung von CrisisChecklisten auf die Leistungsfähigkeit der Gruppe in Notfallsituationen noch nicht systematisch untersucht worden.

\section{Hindernisse für die Einführung von Checklisten unter klinischen Bedingungen}

Die Einführung von Checklisten stößt in der klinischen Routine trotz der für Sicherheitschecklisten gezeigten positiven Effekte in nicht seltenen Fällen auf Skepsis. Vor allem wird bezweifelt, dass eine Crisis-Checkliste mit den ihr inhärenten Limitationen bezüglich der Ausführlichkeit und der Übersichtlichkeit den komplexen Charakter einer Notfallsituation adäquat widerspiegeln kann [25].

Auch kulturelle Eigenheiten im Selbstverständnis von Medizinern stehen einer ähnlich weiten Verbreitung von CrisisChecklisten in der Medizin wie in anderen sicherheitssensiblen Bereichen entgegen. Während in der Luftfahrt der Gebrauch von Crisis-Checklisten in Notfallsituationen von Beginn der Ausbildung an als selbstverständlich gelehrt wird, bildet die therapeutische Freiheit des Arztes in der klinischen Ausbildung und im Selbstverständnis der Ärzteschaft eine feste Größe. Nicht selten wird eine Checkliste hier als Verzicht auf eben jene Therapiefreiheit in kritischen Situationen zugunsten von übersimplifizierten, hoch standardisierten Abläufen missverstanden und als Kritik an der eignen klinischen Kompetenz aufgefasst [25, 26].

Auch die in der Medizin und insbesondere in der Chirurgie noch verbreiteten vertikalen Hierarchien stehen dem Routinegebrauch von Crisis-Checklisten entgegen: Die Versorgung eines kritisch Kranken wird durch ihren Gebrauch zu einer Aufgabe, die vom Team gelöst werden muss und deren checklistenkonforme Ausführung auch im Team überwacht wird; unkontrollierte Autorität ist bei diesem Vorgehen kontraproduktiv. Der Checklistengebrauch führt zu einer Demokratisierung medizinischen Wissens, dessen Umsetzung in der Notfallsituation in einem standardisierten Ablauf von jedem Teammitglied verstanden und nachvollzogen werden kann [27].

\section{Gebrauch von Checklisten in Notfallsituationen: Vorteile und Risiken}

Aus den oben genannten Gründen ergeben sich zahlreiche theoretische Gründe, die nahelegen, dass der Gebrauch von Crisis-Checklisten zu einer Verbesserung der Patientenversorgung führen kann. Klinische Entscheidungen und ihre technische Umsetzung müssen im Rahmen von Notfallsituati- 
Tab. 2. Vorteile und Risiken von Crisis-Checklisten

\begin{tabular}{ll}
\hline Vorteile & Risiken \\
\hline $\begin{array}{l}\text { Zügige Implementierung aktueller Empfehlungen } \\
\text { und Richtlinien in der Praxis }\end{array}$ & $\begin{array}{c}\text { Zementierung veralteter Abläufe bei fehlender } \\
\text { regelmäßiger Überarbeitung }\end{array}$ \\
$\begin{array}{l}\text { Standardisierte Handlungsabläufe } \\
\text { Reduktion von Fehlern durch Unterlassen } \\
\text { Demokratisierung medizinischen Wissens im } \\
\text { Team }\end{array}$ & $\begin{array}{c}\text { Behandlungswege in der aktuellen } \\
\text { klinischen Situation }\end{array}$ \\
$\begin{array}{l}\text { Reduktion von Stress und dessen negativer } \\
\text { Auswirkungen auf die Kognition }\end{array}$ \\
$\begin{array}{l}\text { Verbesserung des Zusammenspiels im Team } \\
\text { bei regelmäßigem Training }\end{array}$ \\
\hline
\end{tabular}

onen in der Regel schneller als im elektiven Setting erfolgen, und dies meist bei höherer technischer Schwierigkeit und gleichzeitig begrenzten technischen Möglichkeiten. Das Vorgehen nach einer standardisierten Crisis-Checkliste erlaubt es hier, Entscheidungszeit zu sparen, Fehler durch Unterlassen zu vermeiden und Einschränkungen der Kognition in Krisensituationen auszugleichen.

Die Versorgung von Notfallpatienten ist immer eine Teamaufgabe. In der klinischen Routine sind feste Teams in konstanter Zusammensetzung aber heute leider die Ausnahme. Gründe hierfür sind die Implementierung des Arbeitszeitgesetzes und zunehmend größere Funktionseinheiten mit häufig wechselnder Teamzusammensetzung. Um trotzdem ein optimales Zusammenspiel in Krisensituationen zu erreichen, ist ein regelmäßiges Teamtraining von Handlungsabläufen, in dem standardisierte Handlungsabläufe mit klar festgelegten Verantwortlichkeiten möglichst unter realistischen Bedingungen trainiert werden können, in einer Krisensituation unerlässlich. Crisis-Checklisten sind hier ein wertvolles Werkzeug, um standardisiert und reproduzierbar festzulegen, wer in welchem Fall wann für welche Maßnahmen verantwortlich ist. Auf dieser Basis können dann standardisierte Abläufe trainiert werden, wie es beispielsweise im ATLS(Advanced Trauma Life Support)- oder ACLS(Advanced Cardiac Life Support)-Konzept bereits praktiziert wird.

Allerdings bergen Crisis-Checklisten auch Gefahren. So ist es wichtig, dass der situationskonforme Gebrauch der Checkliste während der Notfallsituation regelmäßig reevaluiert wird. Sollten neue Informationen erneute differentialdiagnostische Abwägungen erfordern oder patientenspezifische Risikofaktoren das Befolgen des Checklistenalgorithmus als zu risikoreich erscheinen lassen ist, es Aufgabe des Teamleiters, aber auch eines jeden Teammitglieds, den Gebrauch der Checkliste an die jeweilige klinische Situation anzupassen bzw. auf die situationsadäquatere Crisis-Checkliste zu wechseln.

Ein regelmäßiges Überarbeiten der Checkliste und das Anpassen an die Entwicklung des aktuellen Kenntnisstands sind zwingend notwendig und durchaus aufwendig. Der Gebrauch veralteter Checklisten kann dazu führen, dass ein nicht dem State of the Art entsprechender Behandlungsalgorithmus institutionalisiert wird. Die Nutzer müssen über er- folgte Veränderungen informiert und mit diesen Aktualisierungen im Teamtraining vertraut gemacht werden. Nur durch diesen relativ großen Aufwand kann die Checkliste dauerhaft effektiv sein.

Die Vor- und Nachteile von Crisis-Checklisten sind in Tabelle 2 zusammengefasst.

\section{Crisis-Checklisten in der klinischen Routine: Erst wenige Daten verfügbar}

Die Verwendung hoch standardisierter Protokolle in der Notfallmedizin - wie beispielsweise des ATLS- und ACLSKonzepts - führte zu einer nachgewiesenen Verbesserung der Patientenversorgung [28, 29]. Der Einfluss des Gebrauchs von Crisis-Checklisten auf das Management von Notfällen im OP und in der Endoskopie ist heute dagegen erst sehr wenig untersucht. Die Pubmed-Suche unter den oben genannten Stichwörtern liefert erstaunlicherweise nur eine Originalarbeit, die sich mit der Implementierung von Crisis-Checklisten im OP befasst. Die Autoren dieser Studie aus der Arbeitsgruppe um Gawande [6] schätzen, dass bei jährlich 234 Millionen ausgeführten Operationen ca. 3 Millionen intraoperative Notfälle auftreten werden.

Für die Versorgung einer Vielzahl von Notfallsituationen, beispielsweise des akuten Herz-Kreislauf-Stillstands, wurden hoch standardisierte Handlungsempfehlungen (ACLS) entwickelt. Trotzdem konnte konstatiert werden, dass es bei $61 \%$ der unter Simulatorbedingungen mit der Versorgung von lebensbedrohlichen Zwischenfällen befassten Anästhesisten zu erheblichen Abweichungen von diesen Standards kam und dass sichere Kenntnisse in der Notfallversorgung mit zunehmendem Abstand zur klinischen Ausbildung abnehmen [30-33]. Ausgehend von der Feststellung, dass es durch den Gebrauch von Crisis-Checklisten in der Anästhesiologie gelungen war, anästhesiologische Zwischenfälle besser zu versorgen, untersuchten die Autoren den Einfluss von CrisisChecklisten auf die Vorsorgung von Notfällen im OP [6]. Sie entwickelten 12 Checklisten (10 Checklisten für spezifische lebensbedrohliche Krankheitsbilder und 2 Checklisten für symptomorientierte lebensbedrohliche Zustände) (Tab. 3). 
Tab. 3. Themen der von Ziewacz et al. [6] verwendeten Crisis-Checklisten

Anaphylaxie

Feueralarm

Herzstillstand durch Bradykardie/pulslose elektrische Aktivität

Herzstillstand durch Kammerflimmern

Hämorrhagie

Hypotonie

Hypoxie

Kreislaufinstabile Bradykardie

Kreislaufinstabile Tachykardie

Luftembolie

Maligne Hyperthermie

Schwieriger Atemwegszugang

Die Entwicklung der Checklisten erfolgte basierend auf einer umfangreichen Literatursuche, unter Verwendung der aktuellen evidenzbasierten Handlungsempfehlungen und unter Beteiligung von Chirurgen, Anästhesisten, OP-Koordinatoren, Krankenschwestern, Simulationsexperten und eines Checklistenentwicklers der Boeing-Flugzeugwerke. Der Einfluss des Checklistengebrauchs auf das Management kritischer Ereignisse wurde unter Simulatorbedingungen an zwei chirurgischen Teams getestet. Gemessen wurde die von den Teilnehmern subjektiv empfundene Effizienz des Checklistengebrauchs in der Krisensituation. Als eine mehr auf den klinischen Erfolg des Checklistengebrauchs hinweisende Messgröße wurde weiterhin die korrekte Ausführung von spezifischen Schlüsselschritten durch die Probandenteams bewertet.

Es konnte gezeigt werden, dass das subjektive Sicherheitsgefühl der Probanden durch die Checklisten gefördert und der Gebrauch der Checklisten in der Klinik nach der Simulation durch die Probanden befürwortet wurde. Die Zustimmung zum klinischen Checklistengebrauch vor der Simulation wurde nicht erfasst, ebenso wie Einflüsse auf das Stressempfinden der Probanden durch z.B. die Messung von Cortisolspiegeln. Andererseits konnte gezeigt werden, dass der $\mathrm{Ge}$ brauch der Checklisten zu signifikant weniger Abweichungen von den vorher definierten Schlüsselschritten im Krisenmanagement führte. Wie aus dem Studiendesign abzuleiten war, wurden keinerlei harte Daten zur Verbesserung der klinischen Outcomes durch den Checklistengebrauch erhoben.

\section{Schlussfolgerungen}

Unvorhergesehene Zwischenfälle im OP und in der Endoskopie sind in der Regel zeitkritische Ereignisse, die mit einer erheblichen psychischen und teilweise physischen Belastung des versorgenden medizinischen Teams einhergehen. Die damit einhergehende Stressbelastung führt meist $\mathrm{zu}$ einer deutlichen Veränderung der kognitiven Fähigkeiten und stellt zudem hohe Anforderungen an das Zusammenspiel der Beteiligten im Team. In Korrelation zu anderen sicherheitsrelevanten Bereichen (z.B. Luftfahrt und Atomindustrie) sowie $\mathrm{zu}$ von der Verhaltensforschung und Psychologie gelieferten theoretischen Überlegungen lässt sich ableiten, dass der Gebrauch von Crisis-Checklisten in derartigen Situationen zu einer signifikanten Verbesserung der Abläufe führen könnte. Der Nutzen von Crisis-Checklisten in der klinischen Routine kann sich aber nur anhand eines Vorteils für das PatientenOutcome in klinischen Notfällen messen lassen. Dieser Beweis steht nach gegenwärtiger Datenlage trotz der zahlreichen theoretischen Vorteile des Gebrauchs von Crisis-Checklisten bisher aus. Nur durch diese harten klinischen Daten wird sich aber das an der Patientenversorgung beteiligte medizinische Personal von den Vorteilen der Checkliste und der Notwendigkeit ihres Einsatzes in der klinischen Routine überzeugen lassen. Dass diese Untersuchungen sehr anspruchsvoll und aufwendig sind, steht angesichts der Heterogenität der Gruppe der Notfallpatienten außer Frage. Hier ist dringend weitere, klinisch orientierte Forschung notwendig.

\section{Disclosure Statement}

Die Autoren erklären, dass kein Interessenkonflikt vorliegt.

\section{Literatur}

1 Gawande A: The checklist: if something so simple can transform intensive care, what else can it do? New Yorker 2007;86-101.

2 de Vries EN, Ramrattan MA, Smorenburg SM, Gouma DJ, Boermeester MA: The incidence and nature of in-hospital adverse events: a systematic review. Qual Saf Health Care 2008;17:216-223.

3 Committee on Quality of Health Care in America; Institute of Medicine: To Err Is Human: Building a Safer Health System. Washington, DC, The National Academies Press, 2000

4 Walker IA, Reshamwalla S, Wilson IH: Surgical safety checklists: do they improve outcomes? Br J Anaesth 2012;109:47-54.
5 Busemann A, Schreiber A, Heidecke C: Einführung von Operationschecklisten als Teil des Risikomanagements. Chirurg 2012;83:611-616.

6 Ziewacz JE, Arriaga AF, Bader AM, et al: Crisis checklists for the operating room: development and pilot testing. J Am Coll Surg 2011;213:212-219. 7 Schull MJ, Ferris LE, Tu JV, Hux JE, Redelmeier DA: Problems for clinical judgement: 3 . Thinking clearly in an emergency. CMAJ 2001;164:1170-1175.

8 Burian BK: Aeronautical Emergency and Abnormal Checklists: Expectations and Realities. Proceedings of the Human Factors and Ergonomics Society 50th Annual Meeting, San Francisco, 2006. http://human-factors.arc.nasa.gov/publications/ Burian_EAChecklists_Expect_Realities.pdf.
9 Aviation Safety Reporting System: Accession \# 437830. Search Request number 6133. Moffett Field, CA, 2001

10 Transportation Safety Board of Canada: Aviation Investigation Report A98H0003. In-Flight Fire Leading to Collision with Water, Swissair Transport Limited McDonnell Douglas MD-11 HBIWF, Peggy's Cove, Nova Scotia 5 nm SW, 2 September 1998. Gatineau, QC, TSB of Canada, 2003.

11 Selye H: A syndrome produced by diverse nocuous agents. 1936. J Neuropsychiatry Clin Neurosci 1998;10:230-231. 
12 LeBlanc VR: The effects of acute stress on performance: implications for health professions education. Acad Med 2009;84(suppl):S25-33.

13 Chajut E, Algom D: Selective attention improves under stress: implications for theories of social cognition. J Pers Soc Psychol 2003;85:231-248.

14 Bohnen N, Houx P, Nicolson N, Jolles J: Cortisol reactivity and cognitive performance in a continuous mental task paradigm. Biol Psychol 1990;31:107-116.

15 Vedhara K, Hyde J, Gilchrist ID, Tytherleigh M, Plummer S: Acute stress, memory, attention and cortisol. Psychoneuroendocrinology 2000;25:535-549.

16 Buchanan TW, Tranel D, Adolphs R: Impaired memory retrieval correlates with individual differences in cortisol response but not autonomic response. Learn Mem 2006;13:382-387.

17 Lupien SJ, Gillin CJ, Hauger RL: Working memory is more sensitive than declarative memory to the acute effects of corticosteroids: a dose-response study in humans. Behav Neurosci 1999;113:420-430.

18 Beilock SL, Carr TH: On the fragility of skilled performance: what governs choking under pressure? J Exp Psychol Gen 2001;130:701-725.

19 LeBlanc VR, MacDonald RD, McArthur B, King $\mathrm{K}$, Lepine T: Paramedic performance in calculating drug dosages following stressful scenarios in a human patient simulator. Prehosp Emerg Care 2005;9:439-444.
20 Keinan G: Decision making under stress: scanning of alternatives under controllable and uncontrollable threats. J Pers Soc Psychol 1987;52:639-644.

21 Weingart SN, Wilson RM, Gibberd RW, Harrison B: Epidemiology of medical error. BMJ 2000;320: 774-777.

22 Donchin Y, Gopher D, Olin M, et al: A look into the nature and causes of human errors in the intensive care unit. Crit Care Med 1995;23:294-300.

23 Fonne VM, Myhre G: The effect of occupational cultures on coordination of emergency medical service aircrew. Aviat Space Environ Med 1996;67: 525-529.

24 Cannon-Bowers JA, Salas E: Reflections on shared cognition. J Org Behav 2001;22:195-202.

25 Cabana MD, Rand CS, Powe NR, et al: Why don't physicians follow clinical practice guidelines? A framework for improvement. JAMA 1999;282: 1458-1465.

26 Colon-Emeric CS, Lekan D, Utley-Smith Q, et al: Barriers to and facilitators of clinical practice guideline use in nursing homes. J Am Geriatr Soc 2007;55:1404-1409.
27 Winters BD, Gurses AP, Lehmann H, Sexton JB Rampersad CJ, Pronovost PJ: Clinical review: Checklists - translating evidence into practice. Crit Care 2009;13:210

28 van Olden GD, Meeuwis JD, Bolhuis HW, Boxma $\mathrm{H}$, Goris RJ: Clinical impact of advanced trauma life support. Am J Emerg Med 2004;22:522-525.

29 Dane FC, Russell-Lindgren KS, Parish DC, Durham MD, Brown TD: In-hospital resuscitation: association between ACLS training and survival to discharge. Resuscitation 2000;47:83-87.

30 Kurrek MM, Devitt JH, Cohen M: Cardiac arrest in the OR: how are our ACLS skills? Can J Anaesth 1998;45:130-132.

31 Semeraro F, Signore L, Cerchiari EL: Retention of CPR performance in anaesthetists. Resuscitation 2006;68:101-108.

32 Lipman SS, Daniels KI, Carvalho B, et al: Deficits in the provision of cardiopulmonary resuscitation during simulated obstetric crises. Am J Obstet Gynecol 2010;203:179.e1-5.

33 Stross JK: Maintaining competency in advanced cardiac life support skills. JAMA 1983;249:3339_ 3341. 